\title{
El Tutor Universitario como Impulsor del Aprendizaje Reflexivo de los Alumnos Durante las Prácticas Docentes*
}

\author{
University Tutors as Facilitators of Student-teachers' Reflective Learning \\ During their Teaching Practice
}

\author{
Ángeles Diez-Fernández ${ }^{a}$, Raquel Domínguez-Fernández ${ }^{b}$ \\ a Universidad de León, Telf.: (34) 987293030 \\ Correo electrónico: madief@unileon.es

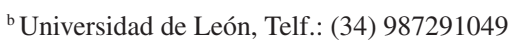 \\ Correo electrónico: rdomf@unileon.es
}

\begin{abstract}
RESUMEN
En el sistema español de prácticas el alumnado percibe que sus tutores universitarios, aunque cumplen satisfactoriamente su rol de mediadores y calificadores, carecen de un papel formativo relevante durante las mismas. El objetivo de este trabajo es analizar esta percepción tras su participación en un modelo de prácticas reflexivo en el que los tutores se plantearon contribuir significativamente a la mejora de las competencias profesionales de sus tutorandos. Con este propósito se utilizó en un postgrado de formación de profesorado de Educación Secundaria un sistema de andamiaje ad hoc basado en técnicas de modelado, aprendizaje cooperativo y evaluación formativa. Mediante un cuestionario se recogieron las valoraciones de los alumnos sobre el papel de este tutor en el estímulo de una serie de acciones reflexivas y sobre el propio procedimiento. Los resultados muestran que, gracias a haber incentivado su reflexión, los postgraduados percibieron a sus tutores universitarios como agentes formativos eficaces.
\end{abstract}

Palabras claves: prácticum, evaluación formativa, reflexión, supervisor, andamiaje.

\begin{abstract}
Within the Spanish teaching practice system students perceive that, despite fulfilling their role as mediators and graders successfully, university tutors do not perform a successful formative role. The objective of this work was to analyze students' perceptions after their participation in a reflective practicum model in which tutors attempted to contribute significantly to the improvement of their students' professional competencies. For this purpose, an ad hoc scaffolding system based on modelling, cooperative techniques, and formative assessment was used in a graduate-level secondary teacher training program. Subsequently, the students answered a questionnaire including items about their university tutors' support in several reflective practices and about the procedure itself. The results showed that, having facilitated their reflection, the students perceived their university tutors as efficient formative agents.
\end{abstract}

Keywords: practicum, formative assessment, reflection, supervisor, scaffolding.

Este trabajo ha sido financiado por la Universidad de León (España) como parte del Plan Anual de Innovación Docente 2016. Las autoras pertenecen al Grupo de Innovación Docente IFAHE de dicha universidad. 


\section{INTRODUCCIÓN}

\subsection{EL PRÁCTICUM REFLEXIVO EN LA FORMACIÓN DEL PROFESORADO}

Los diferentes modelos de prácticas pre-profesionales que se desarrollan en la actualidad tienen como objetivo conseguir la integración del saber disciplinar de las materias de los grados y posgrados correspondientes, el conocimiento metodológico de las mismas y la práctica profesional. Uno de los modelos más valorados es el reflexivo, que considera la reflexión sobre la praxis como el detonante más valioso del aprendizaje del profesional en prácticas. Este se implica en una experiencia vicaria o real, por observación o intervención, y, tras un ejercicio de introspección, inicia un periodo de generalización y preparación para la próxima práctica (Zabalza, 2011).

Cuando se utiliza este modelo para la formación del profesorado, el objetivo central es que los futuros docentes sean capaces de superar la repetición de respuestas elegidas de una lista descontextualizada de soluciones (Weber, 2013) y de razonar sobre los motivos por los que utilizan determinadas estrategias didácticas. Esta capacidad reflexiva les ayudará a detectar situaciones problemáticas, a indagar sobre sus causas y a decidir cuál es la mejor solución a corto y largo plazo (Lee, 2005). Para ofrecer una respuesta adecuada, los graduados o posgraduados tienen que implicarse en un trabajo de análisis y de síntesis (Dewey, 1938 Cit. en Gefulso \& Dennis, 2014), es decir, deben identificar y entender primero y, después, explicar(se) las tensiones entre los elementos involucrados y los dilemas que implican para el docente. Además, para evitar que las prácticas se conviertan en una inmersión acrítica (Pérez, 1997) han de utilizar el conocimiento teórico adquirido durante la formación académica.

Una de las ventajas que aporta la práctica reflexiva es aumentar la eficacia formativa de los docentes, mejorando el aprendizaje de sus alumnos (Sellars, 2012; Weber, 2013). Este modelo convierte a los futuros maestros y profesores en expertos 'flexibles', los adaptive experts que proponen Hammerness, Darling-Hammond \& Bransford (2005), capaces de adaptar sus estrategias a alumnos diferentes en distintos contextos espaciales y temporales, con mejor disposición y posibilidades de modificar la práctica docente. Contribuye, por lo tanto, a la construcción de la identidad profesional del profesor, que comienza a forjarse en la transición de la figura de estudiante a la de docente que se produce durante las prácticas (Flores \& Day, 2006; Stingu, 2012).

Por otra parte, los docentes reflexivos desarrollan la habilidad de generar nuevos saberes tras adaptar su conocimiento teórico y práctico previo para responder a situaciones nuevas (Hobbs, 2011). Este 'nuevo' conocimiento generará procesos innovadores, imprescindibles para preparar a los estudiantes para un mundo que se está continuamente reinventando a sí mismo (Schön, 1983; Domingo \& Gómez, 2004; Sellars, 2012).

\subsection{DIFICULTADES DE LA ENSEÑANZA DE LA REFLEXIÓN EN EL PRÁCTICUM}

Aunque el modelo de prácticum reflexivo está muy extendido, sigue presentando dificultades a la hora de su aplicación. Con frecuencia, los futuros docentes realizan sus observaciones y/o intervenciones en el aula sin apoyo para reflexionar y sintetizar lo que están experimentando y sin la capacidad de vincular el aprendizaje con la teoría (Blanco \& Latorre, 2008). El resultado es que, aunque siempre alcancen un cierto grado de aprendizaje, 
suelen permanecer en el nivel técnico del pensamiento crítico. Sus reflexiones en este nivel tienden a ser descriptivas y están desvinculadas por completo de un marco teórico (Larrivee, 2008; Stingu, 2012; Weber, 2013).

Precisamente, debido a la complejidad que implica la adquisición de estrategias cognitivas de orden superior, muchas de las propuestas de mejora de los programas de formación del profesorado explicitan la conveniencia de incluir, desde un principio, los marcos conceptuales y las destrezas necesarias para la práctica reflexiva crítica en el aula (Banoobhai, 2012; Stingu, 2012; Svojanovski, 2017). Este problema se agrava por la dificultad para encontrar espacios de comunicación eficaces entre los agentes implicados, de ahí que se imponga la necesidad de diseñar e implementar un procedimiento que permita cumplir simultáneamente y con la mayor eficacia los fines del prácticum.

Ya que los alumnos en prácticas deberían recibir un feedback inmediato y constructivo que les permita valorar lo que han aprendido hasta el momento y conocer cuál es la forma más adecuada de mejorar, el sistema de evaluación formativa se presenta como un sistema a priori adecuado para enseñar a reflexionar a los alumnos (Gareis, 2007; López-Pastor \& Pérez-Pueyo, 2017) en las prácticas docentes. Para Gronlund (2006) el objetivo del sistema de evaluación formativa es monitorizar el aprendizaje de los estudiantes durante la instrucción para identificar sus fortalezas y debilidades y poder modificar así tanto la forma de enseñanza como el aprendizaje. Gareis (2007) añade que su fin último es conseguir que los estudiantes puedan autoevaluarse, es decir, que sean capaces de monitorizarse, responder y dirigir su propio aprendizaje tanto dentro como más allá del aula. Así pues, convendría que fuese dentro de los sistemas de mediación puestos en marcha en el entorno académico donde los futuros docentes aprendiesen y desarrollasen las competencias necesarias que les permitieran involucrarse y optimizar los procesos reflexivos estimulados durante sus prácticas en los centros educativos (Larrivee, 2008).

\subsection{EL PAPEL DEL TUTOR UNIVERSITARIO}

En muchos contextos -entre ellos, el español- los prácticum desarrollados en los centros educativos se diseñan por las instituciones educativas a partir de relaciones triádicas que implican al alumno, al tutor del centro (TC) y al tutor universitario o académico (TU) ${ }^{1}$ (Cuenca, 2010; Domínguez et al., 2015). Debido a su relación directa, intensa e ininterrumpida con el alumno durante su estancia en el centro, el TC se ha convertido en el foco de numerosas investigaciones (Cid, Pérez \& Sarmiento, 2011; Martínez \& Raposo, 2011). Cuenca (2010) argumenta que, por el contrario, la figura del TU, que sirve de puente entre el centro escolar y la universidad, ha recibido una menor atención en la literatura sobre la formación del profesorado y, cuando se ha abordado, se ha detectado que, en muchas ocasiones, su contribución a la formación de los tutorandos en esta etapa es escasa, bien por su limitado contacto con el alumno o por su falta de preparación para el rol desempeñado.

La fuerte dependencia del contexto, con responsabilidades que abarcan los diferentes aspectos de la concepción, diseño y especificación de la supervisión (Andreucci, 2013) y dependen, por lo tanto, del plan de prácticas de la universidad (Martínez, 2007), está en

La falta de consenso comienza por su denominación, que es tanto supervisor (o supervisor universitario) (Andreucci, 2013; Blanco \& Latorre, 2008; Cebrián, 2011; Cid et al., 2011; Montecinos, Walker \& Maldonado, 2015) como, en menor medida, tutor universitario -el utilizado en este trabajo- (o tutor académico) (Coiduras et al., 2014; Higgins et al., 2013; Vilá, Aneas \& Rajadell, 2015). 
el origen de la variedad y complejidad de funciones atribuida a los TU (Correa, 2009). Muchos autores (Slick, 1998; Martínez, 2007) coinciden en funciones derivadas de la colaboración y comunicación con el tutor del centro (funciones psicosociales de apoyo) y en la evaluación del documento solicitado a los alumnos (memoria, portafolio). Otros (Cid \& Ocampo, 2007; Coiduras et al., 2014; Cuenca, 2010; Pérez, 2005) añaden a éstas la de orientar al estudiante para que realice unas prácticas reflexivas, trabajando dentro de enfoques constructivistas que permitan la autorregulación del alumno, que fomenten la colaboración y la investigación, y que valoren la capacidad del alumno de reflexionar y evaluar el proceso y los resultados de su propia enseñanza y aprendizaje (Quick \& Dasovich, 1994). Para Zabalza (2011) "la experiencia vivida, por rica y estimulante que sea, puede quedar en nada si no va acompañada de una adecuada supervisión que oriente la reflexión, que ayude a ir más allá de los componentes emocionales de la experiencia, que acompañe los aprendizajes" (p. 33).

En la práctica, la literatura muestra disparidad entre la percepción de los TU y sus alumnos en cuanto a incentivar sus reflexiones. Así, por ejemplo, las investigaciones de Martínez (2007) y Pérez y Latorre (2007) muestran los mismos resultados: mientras sus tutores en la universidad consideran que sí les habían estimulado a reflexionar, "los estudiantes consideraron que sus supervisores no les habían fomentado la reflexión" (Martínez, 2007, p. 144). Para Pérez (2001 Cit. en Molina et al., 2004), ni ayudan a los alumnos a elaborar propuestas didácticas personales, ni les estimulan a buscar las de otros a través de la lectura, ni los hacen críticos con los modelos pedagógicos experimentados.

Estos consideran que los TU, si bien sirven de agentes efectivos en su relación con los centros, fallan en el impulso a la reflexión, necesaria para incrementar su aprendizaje durante las prácticas docentes. Parece entonces necesario diseñar e implementar nuevos procedimientos de tutorización desde la universidad, que permitan a los posgraduados entender y valorar la importancia del papel de sus tutores como impulsores de procesos reflexivos complejos dirigidos a la mejora de sus competencias profesionales.

\subsection{OBJETIVOS E HIPÓTESIS}

En este trabajo se aborda la valoración de los TU por sus alumnos tras una experiencia mediada de aprendizaje basada en la enseñanza reflexiva, diseñada e implementada para la asignatura Prácticum del Máster Universitario en Formación del Profesorado de Educación Secundaria Obligatoria y Bachillerato, Formación Profesional y Enseñanzas de Idiomas (MUFPES) ${ }^{22}$ de la Universidad de León (ULE), España, desarrollada en las sesiones de tutoría realizadas en la universidad y gestionada a través de un portafolio reflexivo. Con este propósito se realizaron las intervenciones, en las aulas universitarias, para mejorar la capacidad reflexiva de los alumnos durante sus prácticas docentes. El fin último fue incentivar su aprendizaje en los centros escolares y optimizar su adquisición de las competencias docentes. Igualmente, se intentó descubrir si el modelo propuesto había servido para que los futuros profesores percibieran que el TU había superado sus funciones tradicionales de mediador y calificador para convertirse, además, en impulsor del aprendizaje reflexivo.

Los objetivos secundarios de esta investigación fueron los siguientes:

En España la realización de este Máster es obligatoria para ejercer la función docente en Enseñanza Secundaria. 
Identificar las expectativas de los estudiantes sobre el papel del TU durante sus prácticas docentes.

Analizar la consideración de los alumnos sobre la contribución del TU a la mejora de sus procesos reflexivos.

Establecer el grado de satisfacción de los alumnos sobre el procedimiento empleado por el TU.

La hipótesis de partida fue que los TU pueden estimular la reflexión del alumnado durante sus prácticas docentes mediante el diseño y la aplicación de un andamiaje y un procedimiento de evaluación formativa ad hoc durante las tutorías universitarias.

\section{METODOLOGÍA}

\subsection{PARTICIPANTES}

En esta experiencia participaron los 44 alumnos del MUFPES matriculados en los módulos de Geografía e Historia (25) y de Inglés (19) durante el curso 2016-2017. Realizaron sus prácticas durante 200 horas (10 ECTS) entre los meses de marzo y mayo en 26 centros de Educación Secundaria: 9 en la ciudad (8 alumnos de Inglés y 13 de Geografía e Historia), en la Escuela Oficial de Idiomas (1 de Inglés), en 7 centros rurales de la provincia (todos de Geografía e Historia), en 6 centros de provincias limítrofes ( 2 de Inglés y 4 de Geografía e Historia), y en 3 centros extranjeros (Portugal, Rumanía y México) a través de los convenios internacionales de la universidad (5 de Inglés y 1 de Geografía e Historia). Son alumnos que cursan por primera vez la asignatura y que tienen una edad media de 24 años.

Durante sus prácticas acompañaron al TC asignado durante todo su horario lectivo, incluyendo -cuando era posible- actividades fuera del aula (reuniones de departamento o con padres, sustituciones) y combinando observación con intervención en función de la disposición de ambos.

Su seguimiento en la ULE se articuló en torno a las tutorías presenciales (para los que podían acudir a la universidad) u on-line (para quienes estaban realizando el prácticum fuera de la provincia), entendidas en la misma línea que indican Zabalza y Cid (2005), como elemento central del prácticum, y dirigidas por dos profesoras tutoras especialistas en las materias supervisadas, ambas con 8 años de experiencia como TU en el MUPFES.

\subsection{PROCEDIMIENTO}

El proceso se secuenció en cuatro fases, con objetivos claramente definidos, abordadas en 5 sesiones (9 horas presenciales):

FASE 1. Comunicar el modelo formativo. Para ello se partió de las expectativas de los alumnos para abordar las peculiaridades de la asignatura Prácticum, final de su etapa escolar y principio de la profesional. Con este objetivo se procedió a las siguientes acciones:

a) Realización de un estudio exploratorio para conocer las expectativas de los alumnos sobre la función del TU durante las prácticas mediante la técnica cooperativa del folio giratorio (Kagan, 1989). En un único folio los alumnos escribieron sus 
respuestas a la pregunta: ¿Cuáles crees que deben ser las funciones de tu tutor en la universidad durante tus prácticas? El folio giró varias veces para que todos pudieran suscribir (con una cruz) -si las consideraban adecuadas- las propuestas de sus compañeros y/o añadir nuevas funciones.

b) Justificación del modelo de prácticas reflexivas que se iba a desarrollar mostrando a los alumnos diferentes posibilidades formativas. En un gran grupo se describieron y discutieron las ventajas y desventajas de diferentes modelos (artesanales, teóricos, reflexivos) (Ur, 1999). Se abordaron las diferentes perspectivas desde las que se ha interpretado el pensamiento reflexivo y se alentó a los estudiantes a optimizar el aprovechamiento de la asignatura, identificando sus saberes y detectando las necesidades de aprendizaje desde su actuación en los centros, como profesional situado.

c) Presentación del instrumento de evaluación y calificación, el portafolio reflexivo, herramienta claramente identificada en la literatura como facilitadora de la práctica reflexiva (Cebrián, 2011; Lyons, 1999; Rodrigues \& Rodriguez-Illera, 2014), consistente en una recopilación de evidencias de aprendizaje asociadas a las competencias oficiales del Máster. Recurriendo a las fuentes identificadas para la reflexión pedagógica -la experiencia, los referentes teóricos y la tradición (Cornejo, 2011)- se pidió a los alumnos que estructurasen cada evidencia en tres secciones:

Descripción de la experiencia observada o realizada en la que se asienta el aprendizaje adquirido, similar al nivel R1 o de recuerdo de Lee (2005).

Análisis de la experiencia: se recogen las reflexiones sobre el aprendizaje adquirido, utilizando sus conocimientos previos (teóricos y/o prácticos) para explicar las causas y consecuencias de lo sucedido en el centro y la generalización de dicho aprendizaje (niveles de racionalización y reflexión de Lee, 2005).

Propuesta de automejora: el alumno reflexiona sobre sus fortalezas y debilidades en relación con las competencias evidenciadas y propone formas de subsanar las últimas en un futuro gracias a la formación permanente.

FASE 2. Implementar dispositivos formativos para la reflexión que faciliten el aprendizaje complejo. Se diseñaron una serie de sesiones de andamiaje pedagógico "duro" (Saye \& Brush, 2002), que buscaban que el alumnado superase la dificultad que supone para ellos vincular los marcos teóricos con las vivencias prácticas, aprovechando la fuerza del grupo para mejorar el aprendizaje individual:

a) Uso de modelos para la familiarización con el instrumento de reflexión, evaluación y calificación de las prácticas, el portafolio, la estructura de las evidencias que lo componen, y la escala de valoración utilizada para evaluarlo.

b) Simulación para andamiar las destrezas de observación y análisis. Los alumnos, que habían sido divididos en grupos pequeños y a los que se habían asignado diferentes competencias (por ejemplo, descubrir los procesos de interacción en el aula o valorar los materiales utilizados), observaron a varios compañeros mientras llevaban a cabo una tarea simple (un puzle) colaborativa o cooperativamente. Cada grupo describió, analizó, redactó y recibió el feedback de sus pares y del TU sobre los resultados de su observación. 
c) Andamiaje de las propuestas de mejora mediante dos técnicas de aprendizaje colaborativo: la resolución estructurada de problemas y la resolución de problemas por parejas pensando en voz alta (Barkley, Cross \& Major, 2005). Un alumno describió y analizó en voz alta una experiencia personal vinculada con una de las competencias del Máster. El TU le ayudó a identificar sus vacíos de aprendizaje, es decir, aquello que todavía no sabría hacer autónomamente en una situación similar. La reflexión se convirtió en grupal cuando todos sus compañeros repitieron el procedimiento. Posteriormente, tras consultar los recursos de aprendizaje presentados por el TU, los alumnos, en parejas, verbalizaron sus reflexiones.

FASE 3. Orientar el aprendizaje a través de tutorías de evaluación formativa. El andamiaje anterior se optimizó con tres sesiones de evaluación formativa en las que las reflexiones de los alumnos, recogidas en sus evidencias de aprendizaje, se evaluaron mediante una escala de valoración y calificación. Durante la primera tutoría el TU y los compañeros (coevaluación) proporcionaron al alumno feedback de su primera evidencia. En la segunda (optativa) se repitió la heteroevaluación y la autoevaluación, esta vez sobre la primera evidencia corregida. En la tercera y última sesión (obligatoria), la tercera evidencia se sometió también a un proceso de autoevaluación, heteroevaluación y coevaluación (Alonso et al., 2017; Domínguez et al., 2015). En estas tutorías se buscó que los alumnos fuesen conscientes, con el análisis propio y con la ayuda de sus pares y del experto, de cómo podían aprovechar su experiencia para aprender más, explicitando la conexión entre lo que sabían y lo que aún desconocían, pero deberían aprender para ser profesionales más competentes.

FASE 4. Evaluar la percepción del alumnado sobre el modelo formativo seguido en la asignatura utilizando la averiguación por cuestionario. Se intentó que los alumnos valorasen la ayuda explícita proporcionada por el TU para fomentar estrategias que los conviertan en profesionales reflexivos. Para ello, una vez finalizadas las prácticas en los centros, y a la vez que entregaban el portafolio final para su evaluación y calificación, se les pidió que completasen un cuestionario donde respondieron a ítems que buscaban averiguar en qué medida el TU había incentivado o no sus procesos reflexivos y el grado de satisfacción con el procedimiento seguido.

\subsection{INSTRUMENTOS}

Las expectativas y valoraciones de los alumnos fueron recogidas mediante dos instrumentos: el folio giratorio y el cuestionario. El folio giratorio, con el que se trataba de detectar las expectativas de los alumnos sobre las funciones del TU en el prácticum, se usó en la primera sesión de la asignatura, previa a las prácticas.

El cuestionario para medir la satisfacción del alumnado fue completado por estos al concluir la asignatura y está estructurado en dos secciones. La primera, que consta de 22 ítems adaptados del instrumento diseñado por Pérez (2005) para su investigación sobre acciones reflexivas en los prácticum de Magisterio, se utiliza para detectar la apreciación de los alumnos sobre la reflexividad promovida y realizada en la asignatura por los TU. La segunda sección del cuestionario, más breve, consiste en una serie de ocho ítems relativos al modelo utilizado para estimular y mejorar la reflexión de los alumnos sobre sus prácticas a través de las sesiones de tutoría que andamian el aprendizaje y la evaluación formativa del mismo. Las respuestas se recogen mediante una escala Likert, con puntuaciones mínimas de 
1 y máximas de 5, indicando las bajas menor acuerdo con las sentencias enunciadas y las altas un mayor acuerdo según la gradación $1=$ Nada, $2=$ Poco, $3=$ Algo, $4=$ Bastante y $5=$ Mucho.

Los datos del cuestionario se analizaron mediante el software estadístico SPSS (versión 22.0). Se utilizó estadística descriptiva (media y desviación estándar) para calcular las puntuaciones de los diversos ítems del cuestionario. Por otra parte, y dado que las distribuciones de las variables no cumplían en su mayoría supuestos de normalidad, se utilizó estadística no paramétrica ( $U$ de Mann-Whitney) para verificar la existencia de diferencias entre los grupos de Geografía e Historia y de Inglés. El nivel de significación se estableció en $p<0,05$.

\section{RESULTADOS}

En función de las respuestas dadas al comenzar la asignatura, los alumnos esperaban del TU que realizase una serie de funciones: las equivalentes a las dos grandes categorías identificadas para los tutores de los centros, la pedagógica y la psicológica (Martínez \& Raposo, 2011), además de una tercera específica del TU. Estas se pueden concretar en (Gráfico 1):

- Orientar sobre la elaboración del portafolio reflexivo que han de entregar al final de sus prácticas y que constituye el elemento fundamental de la evaluación en la universidad $(39,1 \%)$, con explicitaciones como informar sobre las fechas de entrega $(1,7 \%)$, dar pautas para su elaboración $(19,1 \%)$, solventar todas las dudas que les plantee su realización $(12,2 \%)$ y corregirlo $(6,1 \%)$.

- Estar disponible $(34,8 \%)$ para solucionar cuantas dudas les puedan surgir $(22,6 \%)$ $\mathrm{y}$ atender a sus necesidades $(12,2 \%)$.

- Actuar de mediador con el tutor del centro escolar en el que realizarán las prácticas (26,1\%), con funciones que irían desde ponerse en contacto con el tutor al principio del prácticum $(2,6 \%)$, hacer un seguimiento de la estancia en el centro $(10,4 \%)$ y tratar de solucionar los conflictos que pudieran producirse entre alumno y tutor de centro (13\%).

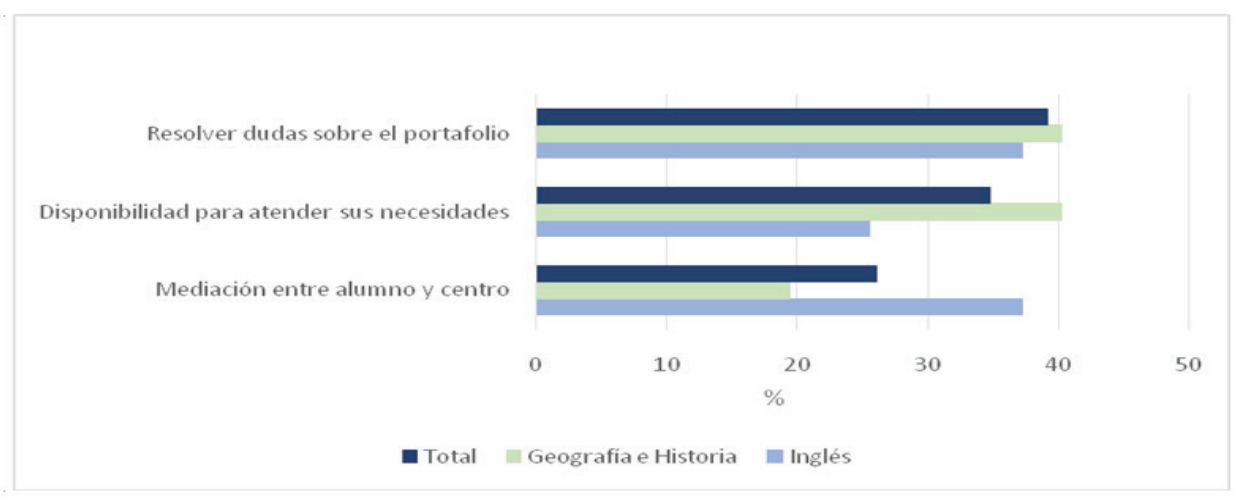

Gráfico1. Expectativas de los alumnos sobre la función del tutor académico 
La principal preocupación de los alumnos de ambos módulos era que el TU les asesorase en la elaboración del portafolio, informando de los procedimientos necesarios para redactarlo y entregarlo, además de corregirlo. En definitiva, el foco estaba puesto en su función evaluadora.

Conviene destacar que, a la par que su papel de evaluador, para los alumnos del módulo de Inglés fue importante la función de mediador con el centro de prácticas, poniéndose en contacto con el mismo, interviniendo en caso de conflicto con el tutorando y haciendo el seguimiento de sus prácticas, mientras que para los de Geografía e Historia, lo fue su disponibilidad para atender sus necesidades individuales y sus dudas (todavía imprecisas).

En cuanto a la percepción de los alumnos, al concluir la asignatura, sobre la contribución del TU al aprendizaje reflexivo y sobre el procedimiento seguido para impulsarlo desde la universidad, ella queda reflejada en la Tabla 1, que muestra los resultados generales de cada una de las preguntas del cuestionario, así como de las dos especialidades estudiadas.

Como se puede apreciar, los participantes manifestaron estar entre bastante y muy de acuerdo con 25 de las 30 preguntas del cuestionario (83,3\%) y entre algo y bastante de acuerdo en las restantes cinco (16,6\%). La comparación entre los grupos de Geografía e Historia y de Inglés mostró diferencias significativas en nueve preguntas (30\%), siempre con la especialidad de Inglés obteniendo puntuaciones más altas excepto en la pregunta 29. "El procedimiento de scaffolding de resolución estructurada de problemas me ha resultado útil para aprender", en la que la especialidad de Geografía e Historia obtuvo puntuaciones significativamente más elevadas.

Tabla 1. Resultados generales de las preguntas del cuestionario

\begin{tabular}{|c|c|c|c|c|c|c|c|}
\hline & \multicolumn{2}{|c|}{ Total } & \multicolumn{2}{|c|}{$\mathrm{G} \& \mathrm{H}$} & \multicolumn{2}{|c|}{ Inglés } & \multirow[b]{2}{*}{ D } \\
\hline & $M$ & $D E$ & $M$ & $D E$ & $M$ & $D E$ & \\
\hline $\begin{array}{l}\text { 1. Me han incentivado a indagar sobre los } \\
\text { diferentes modelos de enseñanza que he } \\
\text { observado. }\end{array}$ & 4,43 & 0,59 & 4,40 & 0,50 & 4,47 & 0,70 & 0,461 \\
\hline $\begin{array}{l}\text { 2. Me han hecho ver la importancia del contexto y } \\
\text { animado a ser flexible. }\end{array}$ & 4,09 & 0,60 & 4,00 & 0,58 & 4,21 & 0,63 & 0,244 \\
\hline $\begin{array}{l}\text { 3. Me han animado a cuestionar los porqués del } \\
\text { currículo, su contenido y su secuencia. }\end{array}$ & 3,86 & 0,82 & 3,64 & 0,76 & 4,16 & 0,83 & $0,026^{*}$ \\
\hline $\begin{array}{l}\text { 4. Me han impulsado a ser crítico con los métodos } \\
\text { y procedimientos de enseñanza-aprendizaje } \\
\text { puestos en práctica. }\end{array}$ & 4,50 & 0,70 & 4,48 & 0,65 & 4,53 & 0,77 & 0,603 \\
\hline $\begin{array}{l}\text { 5. Me han estimulado a examinar críticamente los } \\
\text { valores que se promueven en el aula. }\end{array}$ & 3,64 & 0,92 & 3,32 & 0,80 & 4,05 & 0,91 & $0,008^{*}$ \\
\hline $\begin{array}{l}\text { 6. Me han ayudado a reflexionar sobre mi } \\
\text { actuación. }\end{array}$ & 4,41 & 0,73 & 4,36 & 0,64 & 4,47 & 0,84 & 0,320 \\
\hline $\begin{array}{l}\text { 7. Me han hecho reparar en los efectos que } \\
\text { producen las estrategias de enseñanza- } \\
\text { aprendizaje que utilizo. }\end{array}$ & 4,23 & 0,74 & 4,16 & 0,69 & 4,32 & 0,82 & 0,330 \\
\hline
\end{tabular}




\begin{tabular}{|c|c|c|c|c|c|c|c|}
\hline $\begin{array}{l}\text { 8. Me han animado a ir construyendo mi propio } \\
\text { estilo docente. }\end{array}$ & 4,16 & 0,86 & 4,04 & 0,89 & 4,32 & 0,82 & 0,269 \\
\hline $\begin{array}{l}\text { 9. Me han instado a consultar artículos y a } \\
\text { utilizarlos en mis clases. }\end{array}$ & 4,50 & 0,63 & 4,56 & 0,65 & 4,42 & 0,61 & 0,358 \\
\hline $\begin{array}{l}\text { 10. Me han alentado a resolver mis propias } \\
\text { dificultades como docente. }\end{array}$ & 4,00 & 0,65 & 3,80 & 0,58 & 4,26 & 0,65 & $0,019 *$ \\
\hline $\begin{array}{l}\text { 11. Han favorecido en mí el desarrollo de procesos } \\
\text { reflexivos como forma de aprendizaje. }\end{array}$ & 4,52 & 0,63 & 4,60 & 0,65 & 4,42 & 0,61 & 0,241 \\
\hline $\begin{array}{l}\text { 12. Me han estimulado a recoger información de } \\
\text { mis alumnos sobre la efectividad de la } \\
\text { instrucción. }\end{array}$ & 4,23 & 0,77 & 4,12 & 0,67 & 4,37 & 0,90 & 136 \\
\hline $\begin{array}{l}\text { 13. Me han enseñado a evaluar mi propia actuación } \\
\text { docente. }\end{array}$ & 4,57 & 0,59 & 4,56 & 0,51 & 4,58 & 0,69 & 0,608 \\
\hline $\begin{array}{l}\text { 14. Me han estimulado a hacer planes que mejoren } \\
\text { mi enseñanza. }\end{array}$ & 4,32 & 0,71 & 4,24 & 0,72 & 4,42 & 0,69 & 0,392 \\
\hline $\begin{array}{l}\text { 15. Me han entrenado para generar nuevas ideas } \\
\text { que mejoren mis clases. }\end{array}$ & 4,36 & 0,57 & 4,36 & 0,64 & 4,37 & 0,50 & 0,893 \\
\hline ormular hipótesis sobre mi & 4,07 & 0,66 & 3,92 & 0,57 & 4,26 & 0,73 & 0,080 \\
\hline $\begin{array}{l}\text { 17. Me han hecho comprobar la diferencia entre lo } \\
\text { que es la profesión docente y lo que creía que } \\
\text { era. }\end{array}$ & 3,77 & 0,96 & 3,40 & 0,82 & 4,26 & 0,93 & $0,003 *$ \\
\hline $\begin{array}{l}\text { 18. Me han estimulado a cuestionarme qué hace } \\
\text { que funcionen las cosas, en qué contexto y por } \\
\text { qué. }\end{array}$ & 3,89 & 0,75 & 3,68 & 0,56 & 4,16 & 0,90 & $0,024^{*}$ \\
\hline $\begin{array}{l}\text { 19. Me han estimulado a considerar los principios } \\
\text { éticos y políticos que subyacen a mi actuación } \\
\text { en clase }\end{array}$ & 3,64 & 0,92 & 3,32 & 0,80 & 4,05 & 0,91 & $0,008 *$ \\
\hline $\begin{array}{l}\text { 20. Me han animado a comparar mi pensamiento } \\
\text { sobre la enseñanza con el de otros docentes, } \\
\text { otros compañeros, etc. }\end{array}$ & 4,00 & 0,65 & 3,80 & 0,58 & 4,26 & 0,65 & $0,019 *$ \\
\hline $\begin{array}{l}\text { 21. Me han animado a buscar estrategias para } \\
\text { resolver los problemas que se producen en } \\
\text { clase. }\end{array}$ & 4,23 & 0,74 & 4,16 & 0,69 & 4,32 & 0,82 & 0,330 \\
\hline $\begin{array}{l}\text { 22. Me han estimulado a buscar alternativas para } \\
\text { mi entrenamiento. }\end{array}$ & 4,36 & 0,57 & 4,36 & 0,64 & 4,37 & 0,50 & 0,893 \\
\hline $\begin{array}{l}\text { 23. La evaluación formativa desarrollada me ha } \\
\text { ayudado a aprender. }\end{array}$ & 4,68 & 0,60 & 4,80 & 0,50 & 4,53 & 0,70 & 0,117 \\
\hline $\begin{array}{l}\text { 24. La evaluación formativa desarrollada me va a } \\
\text { ayudar a tener mejores calificaciones. }\end{array}$ & 4,59 & 0,66 & 4,72 & 0,54 & 4,42 & 0,77 & 0,120 \\
\hline $\begin{array}{l}\text { 25. Conocer los indicadores e instrumentos de } \\
\text { evaluación y calificación me va a ayudar a tener } \\
\text { mejores calificaciones. }\end{array}$ & 4,30 & 0,79 & 4,08 & 0,81 & 4,58 & 0,69 & $0,034 *$ \\
\hline $\begin{array}{l}\text { 26. La autoevaluación de las evidencias me ha } \\
\text { ayudado a aprender. }\end{array}$ & 4,27 & 0,79 & 4,40 & 0,58 & 4,11 & 0,99 & 0,465 \\
\hline
\end{tabular}




\begin{tabular}{|l|l|l|l|l|l|l|l|}
\hline $\begin{array}{l}\text { 27. La coevaluación de las evidencias me ha } \\
\text { ayudado a aprender. }\end{array}$ & 4,52 & 0,66 & 4,52 & 0,51 & 4,53 & 0,84 & 0,459 \\
\hline $\begin{array}{c}\text { 28. El procedimiento de scaffolding de uso de } \\
\text { modelos me ha resultado útil para aprender. }\end{array}$ & 4,55 & 0,66 & 4,52 & 0,59 & 4,58 & 0,77 & 0,462 \\
\hline $\begin{array}{c}\text { 29. El procedimiento de scaffolding de resolución } \\
\text { estructurada de problemas me ha resultado útil } \\
\text { para aprender. }\end{array}$ & 4,50 & 0,73 & 4,72 & 0,46 & 4,21 & 0,92 & $0,046 *$ \\
\hline $\begin{array}{c}\text { 30. El procedimiento de scaffolding de resolución } \\
\text { de problemas por parejas pensando en voz alta } \\
\text { me ha resultado útil para aprender. }\end{array}$ & 4,64 & 0,49 & 4,60 & 0,50 & 4,68 & 0,48 & 0,570 \\
\hline
\end{tabular}

G \& H = Geografía e Historia. * $p<0,05$ en la comparación entre las especialidades de Geografía e Historia y de Inglés

\section{DISCUSIÓN}

La experiencia descrita y el análisis de los cuestionarios confirma la hipótesis planteada, ya que los alumnos han percibido que sus TU desempeñan un rol formador dentro del modelo seguido, al que Shön (1987) denomina prácticum reflexivo. Los resultados muestran con claridad el cambio que se produce en la percepción del alumnado sobre el papel de los TU en las prácticas académicas, que pasa de ser considerado mero intermediario entre el centro y la universidad a ser apreciado como agente formativo. Especialmente relevantes son las altas puntuaciones que han otorgado a los TU en la gran mayoría de ítems planteados en el cuestionario (medias superiores a 4), lo que indica un gran acuerdo sobre el estímulo de las acciones reflexivas en sus prácticas y el valor del proceso realizado para alentarlas y mejorarlas. Conviene, además, destacar que se han encontrado algunas diferencias significativas entre los dos módulos analizados.

$\mathrm{Al}$ comienzo de la asignatura las expectativas de los alumnos sobre las funciones del TU coinciden con los roles tradicionales recogidos por la literatura. Uno de ellos es el papel de mediador entre los diferentes agentes de las prácticas, que los posgraduados concretan en la supervisión de las actividades y, sobre todo, en la resolución de conflictos. Henry \& Beasley (1989 Cit. en Quirk \& Dasovich, 1994) explican que los estudiantes buscan un confidente personal, que tanto inspecciona la estancia en los centros como aconseja en los malentendidos y se relaciona de forma individual con cada uno.

En este mismo apartado, nuestros alumnos señalan como función más importante del TU la de evaluador, que ellos confunden con la de -calificador-, mero registrador de un resultado final. Debido a su falta de experiencia previa en la elaboración de un portafolio les preocupa su realización y la calificación final, atribuyendo a su tutor en la universidad parte de la responsabilidad de su éxito o fracaso y demandándole que esté disponible para resolver sus dudas.

Los estudiantes no se plantean en ningún momento que desde la universidad se les pueda ayudar a optimizar su aprendizaje durante las prácticas. Asumen que hay una disociación absoluta entre esta y el centro escolar, por lo que puede anticiparse que el conocimiento académico que han adquirido durante su formación previa va a ser efímero y a desaparecer fagocitado por la supremacía del conocimiento práctico (Pérez, 1997). Consecuentemente, 
para ellos en el momento inicial el TU conserva su papel de actor secundario del prácticum, desprovisto de funciones formativas.

Resulta especialmente significativo el cambio constatado tras las prácticas en la percepción de los alumnos sobre las funciones del TU, que diverge de los resultados obtenidos por otros estudios similares (Martínez, 2007; Pérez \& Latorre, 2007). Han sido conscientes de que les ha guiado en el desarrollo de ciclos de investigación-acción educativa cada vez más complejos y variados en los que, como indica Zeichner (1992), han tenido que confrontar su experiencia en los centros con los conceptos y la teoría, ayudándoles a responsabilizarse de su propio desenvolvimiento profesional.

La actuación del TU se ha percibido por los alumnos como efectiva para superar lo que Pérez (1997) denomina conocimiento pedagógico folklórico y asentar un conocimiento experto y profesional a partir de una reconstrucción crítica del mismo, convirtiéndolos en mediadores activos entre la teoría y la práctica (Edeltesin, 2002). Los posgraduados han advertido el acompañamiento de su TU en el desarrollo de sus competencias profesionales a partir de los elementos de reflexión trabajados. Los datos resultantes del cuestionario mostraron grados muy elevados de acuerdo en ítems que coinciden con los dos primeros niveles de reflexión propuestos por Van Manem (1977), el técnico y el práctico: 4 ("Me han impulsado a ser crítico"), 9 ("Me han instado a consultar artículos"), 11 ("Han favorecido en mí el desarrollo de procesos reflexivos") y 13 ("Me han enseñado a evaluar mi propia actuación docente"). Estos muestran las acciones reflexivas previas a la práctica docente y posteriores a la misma vinculadas con el qué y cómo enseñar, con la actuación docente y con la construcción del estilo docente personal. Los niveles algo más bajos en algunos ítems como el 8 ("Me han animado a ir construyendo mi propio estilo docente"), el 10 ("Me han alentado a resolver mis propias dificultades como docente") o el 20 ("Me han animado a comparar mi pensamiento") sugieren la necesidad de trabajar para que los alumnos comprendan que la identidad profesional de un docente está influida por múltiples factores y no se construye de forma aislada y acrítica. Para superar este prejuicio se propone alentar la colaboración entre profesores y, al mismo tiempo, insistir en la relevancia de su formación previa y continua.

Varios de los ítems relacionados con el que Van Manem (1977) denomina tercer nivel o nivel de reflexión crítica, que se traduce en la incorporación de criterios morales y éticos en el discurso docente (Zeichner, 1992), obtuvieron valores menores. Son sobre todo los alumnos del módulo de Geografía e Historia los que han percibido un menor estímulo en ítems que implican cuestiones morales y éticas, como el 5 ("Me han estimulado a examinar críticamente los valores"), el 17 ("Me han hecho comprobar la diferencia") y el 19 ("Me han estimulado a considerar los principios éticos"). Esta situación puede deberse a que resulta especialmente difícil el desarrollo de procesos reflexivos en este tercer nivel de reflexión por la carga ideológica vinculable al conocimiento disciplinar (Atienza, 2007; Benejam et al., 2002; Souto, 2010; Keršytė, 2017). En todo caso, con niveles más altos de reflexión se podría lograr que los profesores en prácticas mejorasen su capacidad para detectar las relaciones entre el sistema educativo, el centro escolar y la sociedad y prestasen atención a cuestiones como la igualdad o la justicia social (Zeichner, 1990), valorando las consecuencias, tanto éticas como políticas, de sus decisiones.

En cuanto al procedimiento, los alumnos han puesto de manifiesto, a través de los ítems finales del cuestionario (ítems 23 a 30), la satisfacción con el sistema utilizado para estimular y mejorar la reflexión. Así, los estudiantes valoran muy positivamente que el TU haya construido un sistema de evaluación formativa enriquecido con andamiaje previo 
que les oriente en la realización de las tareas complejas, estimulando la necesidad de reflexionar individualmente, explicitando en sus portafolios los aprendizajes adquiridos en los centros de prácticas y propiciando la creación de espacios comunes de reflexión en los que compartir vivencias, con el experto y con los iguales dentro de las tutorías.

En este sentido, los profesores en prácticas han considerado que las técnicas de aprendizaje colaborativo han resultado eficaces para potenciar niveles de reflexión que sobrepasan el nivel individual de la experiencia (Schön, 1992). Los pares han actuado como facilitadores de la práctica reflexiva enriqueciendo los análisis personales (Perrenoud, 2004), mejorando la cantidad y calidad de sus aportaciones a los portafolios, la confianza en sí mismos y, como consecuencia, su autonomía.

Uno de los obstáculos ya detectados por autores como Cornejo (2011) para la mejora de las habilidades reflexivas, que parece ser la explicación para las valoraciones más bajas del ítem 29 en el módulo de Inglés, es la falta de tiempo. La concentración de las prácticas en un periodo tan breve restringe en exceso los momentos disponibles para el andamiaje y las tutorías formativas y concentra la intervención del TU en un espacio tan corto que, ya que el proceso reflexivo no es espontáneo, dificulta que el aprendizaje de los alumnos se asiente de forma adecuada. A pesar de estas dificultades iniciales de tiempo para reunirse y confianza en el procedimiento los estudiantes han acabado valorando muy positivamente la búsqueda de espacios comunes que, como indica Zeichner (1990), refuercen sus lazos, con la creación de verdaderas comunidades de aprendizaje donde los futuros profesores apoyen mutuamente su crecimiento.

Además, los datos analizados refrendan la eficacia de llevar a cabo procedimientos de evaluación formativa en los que los alumnos, en este caso guiados por el TU, adoptan un papel activo como evaluadores de sus evidencias de aprendizaje y de las de sus compañeros (Hamodi, 2016; López-Pastor, 2009, 2011). Se corrobora lo ya planteado por múltiples autores (Álvarez, 2012; Biggs, 2005; Brown \& Glasner, 2003) sobre la relevancia del enfoque evaluador y el sistema de evaluación si queremos garantizar la calidad de los procesos de formación.

A este respecto es relevante el hecho de que los estudiantes hayan corregido su concepción errónea de la evaluación como sinónimo de calificación, transformando su idea inicial del TU como mero evaluador sancionador en la de evaluador formador, en este caso, impulsor del aprendizaje reflexivo. Los propios alumnos han considerado fundamental para este cambio el procedimiento desarrollado, que les ha llevado a entender la evaluación como un proceso de valoración y toma de decisiones cuya finalidad es corregir los errores durante el mismo, ayudando al alumno a aprender más y al profesor a trabajar cada vez mejor (López-Pastor, 2011).

Resulta especialmente interesante el hecho de que las diferencias entre módulos no sean significativas hasta en el $70 \%$ de los ítems planteados, lo cual parece evidenciar que los resultados obtenidos no están principalmente determinados por el estilo personal de los TU o de su alumnado. Así, el establecimiento de una serie de pautas consensuadas y secuenciadas, comunes para los diferentes módulos, se ha logrado desarrollar la asignatura con planteamientos coherentes con el modelo formativo asumido en el MUFPES, respetando las especificidades de cada uno de ellos. Con todo, también existen diferencias que pueden atribuirse por ejemplo al estilo personal del docente, a las características de los alumnos, de las especialidades que cursan o de los centros en los que desarrollan sus prácticas. Estos y otros factores podrían ser objeto de estudio en futuros trabajos. 
Parte del éxito del cambio en las percepciones de los alumnos puede deberse a su implicación en el diseño del proceso, que, consecuente con el modelo de investigación acción planteado en la asignatura, pretende responder a las dificultades planteadas en años anteriores, como la frustración ante la dificultad de la tarea demandada y al aislamiento para resolverla. Igualmente, sería interesante, en ambos módulos, extender el trabajo colaborativo a otros espacios y otros agentes que incluyesen nuevos foros de discusión y otros miembros de la comunidad educativa (profesores de los centros, alumnos de otros módulos), más allá de las tutorías pautadas por los docentes universitarios.

\section{CONCLUSIÓN}

En este trabajo se ha analizado la valoración de los alumnos sobre el papel de sus TU como agentes formativos en la asignatura Prácticum y sobre el procedimiento seguido para alentar la reflexión sobre las experiencias vividas durante sus prácticas, haciendo especial énfasis en el modelo de evaluación. Lo expuesto nos permite concluir que, para los posgraduados del MUPFES, la presencia activa de sus TU ha sido percibida como relevante para conseguir la mejora del aprendizaje adquirido en los centros escolares mediante la utilización de procesos reflexivos. Sin embargo, a nivel práctico en cursos sucesivos se plantea la necesidad de incidir en los niveles de reflexión superiores, llevando a cabo un trabajo más exhaustivo enfocado a la construcción del estilo personal docente y, sobre todo, a la promoción de valores asociados a la profesión.

Al superar su papel burocrático y asumir el de agentes formativos se constata que los TU, además de haber intervenido en la solución de las dificultades surgidas, pueden ayudar a reflexionar sobre los contenidos y procedimientos de las materias, a generar nuevas ideas que contribuyen a la mejora de su actuación en el aula y a la creación del estilo docente personal de sus tutorandos. En este desarrollo resulta clave el diseño e implementación de un procedimiento de estímulo a la reflexión en el que juega un papel muy importante la mayor vinculación del TU con los estudiantes y la reflexión entre pares, reduciendo el aislamiento clásico del alumnado en los centros escolares.

Dado que los alumnos no están entrenados para abordar procesos reflexivos complejos y carecen inicialmente de estrategias suficientes para enfrentarse a la tarea demandada en el sistema de evaluación de las prácticas de los futuros docentes, su andamiaje resulta imprescindible para conseguir buenos resultados. Este aprendizaje ha de realizarse dentro de un plan de tutorías consensuado entre las diversas especialidades del MUFPES, coherente, con contenido propio y que demande la presencia de los alumnos en el aula universitaria como parte de la asignatura de prácticas. Puesto que supone más carga de trabajo para los diferentes agentes implicados, debería integrarse dentro de un plan de prácticas sostenible y organizado a nivel institucional, que permita mejorar y reconocer la intervención de los tutores universitarios, a la vez que homogeneizar los resultados de aprendizaje obtenidos por los alumnos.

En sucesivos trabajos se prevé ampliar la muestra utilizada, incluyendo grupos control, y abrir nuevas vías de investigación complementarias, como el análisis de contenido de las evidencias de los portafolios, el rendimiento académico, la contribución de los tutores de los centros al aprendizaje de los alumnos y la correlación y cohesión de los procedimientos evaluadores de los diferentes agentes implicados: alumnos, tutor de centro y tutor universitario. 
A pesar de los límites planteados, los resultados son de utilidad tanto para la investigación educativa como para la formación de futuros docentes, ya que permiten percibir que el tutor universitario, uno de los agentes de la triada formativa del prácticum, ha superado su tradicional papel secundario y ha adquirido una posición crucial en un modelo formativo reflexivo, contribuyendo a una mayor eficacia en la formación inicial docente.

\section{REFERENCIAS BIBLIOGRÁFICAS}

Alonso, M. D., Diez, M. A., Pérez-Pueyo, A., Domínguez, R., González, I., Fernández, R., . . García, M. E. (2017). La evaluación de competencias y subcompetencias a través de procesos de evaluación formativa con portafolio y rúbricas en el Máster de Formación del Profesorado. En V. López-Pastor y Á. Pérez-Pueyo (Coords.), Evaluación formativa y compartida en educación: experiencias de éxito en todas las etapas educativas (pp. 386-401). León: Servicio de Publicaciones de la Universidad de León. Recuperado de https://buleria.unileon.es/ handle/10612/5999.

Álvarez, J. M. (2012). Pensar la evaluación como recurso de aprendizaje. En B. Jarauta y F. Imbernón (Eds.), Pensando en el futuro de la educación; una nueva escuela para el siglo XXII (pp. 139158). Barcelona: Graó.

Andreucci, P. (2013). La Supervisión de Prácticas Docentes: Una Deuda Pendiente de la Formación Inicial de Profesores. Estudios Pedagógicos, 39(1), 7-26. Recuperado de https://scielo.conicyt. cl/pdf/estped/v39n1/art01.pdf.

Atienza, E. (2007). Discurso e ideología en los libros de texto de ciencias sociales. Discurso \& Sociedad, 1(4), 542-574.

Banoobhai, M. (2012). Critical reflection: tools for curriculum implementation and innovation. Procedia-Social and Behavioural Sciences, 4, 175-179. doi:10.1016/j.sbspro.2012.06.634.

Barkley, E. F., Cross, K. P., \& Major, C. H. (2005). Collaborative learning techniques: A handbook for college faculty. San Francisco: Jossey-Bass.

Benejam, P., Berges, L., CP Joanot Martorell, Grupo Cronos, Hernández, X., Iglesias, J.C., . . Yáñez, M.E. (2002). Las ciencias sociales: concepciones y procedimientos. Barcelona: Grao.

Biggs, J. (2005). Calidad del aprendizaje universitario. Madrid: Narcea.

Blanco, F., \& Latorre, M. J. (2008). La apuesta por la excelencia en la formación práctica universitaria de futuros profesores. Estudios sobre Educación, 15, 7-29. Recuperado de http://hdl.handle. net/10171/9126.

Brown, S., \& Glasner, A. (2003). Evaluar en la universidad. Problemas y nuevos enfoques. Madrid: Narcea

Cebrián, M. (2011). Supervisión con e-portafolios y su impacto en las reflexiones de los estudiantes en el practicum. Estudio de caso. Revista de Educación, 354, 183-208.

Cid, A., Pérez, A., \& Sarmiento, J. A. (2011). La tutoría en el Prácticum. Revisión de la literatura. Revista de Educación, 354, 127-154.

Cid, A., \& Ocampo, C. I. (2007). Funciones tutoriales en el Prácticum de Psicopedagogía en la Universidad de Vigo: percepción de los estudiantes actuales. Revista de Educación, 344, 285307. Recuperado de http://www.revistaeducacion.mec.es/re344_12.html.

Coiduras, J., París, G., Torrelles, C., \& Carrera, X. (2014). La evaluación de competencias en una experiencia de formación dual de maestros: diferencias y semejanzas entre tutores de escuela y de universidad. Estudios pedagógicos, 40, 29-48.

Cornejo, J. (2011). El pensamiento reflexivo entre profesores. Pensamiento Educativo. Revista de Investigación Educacional Latinoamericana, 32(1), 343-373.

Correa, E. (2009). El supervisor de prácticas: recursos para una supervisión. Pensamiento Educativo, 
Estudios Pedagógicos XLIV, N²: 311-328, 2018

EL TUTOR UNIVERSITARIO COMO IMPULSOR DEL APRENDIZAJE REFLEXIVO DE LOS ALUMNOS DURANTE LAS PRÁCTICAS DOCENTES

1(44-45), 237-254.

Cuenca, A. (2010). Care, thoughtfulness, and tact: conceptual framework for university supervisors. Teaching Education, 21(3), 263-278.

Domingo, A., \& Gómez, M. V. (2004). La práctica reflexiva. Bases, modelos e instrumentos. Madrid: Narcea.

Domínguez, R., González, M. I., Fernández, R., Pérez-Pueyo, Á., Gutiérrez, C., Alonso, M. D., ... Diez, A. (2015). Propuesta para una doble evaluación triádica del Máster de Educación Secundaria. En A. Fidalgo, M.L. Sein-Echaluce y F. J. García-Peñalvo (Coords.), La sociedad del Aprendizaje. Actas del III Congreso internacional sobre Aprendizaje Innovación y Competitividad. CINAIC 2015 (16 octubre de 2015, Madrid, España, pp. 565-570). Madrid: Fundación General de la Universidad Politécnica de Madrid. Recuperado de http://www.dmami. upm.es/dmami/documentos/liti/Actas_CINAIC_2015.pdf.

Edelstein, G. E. (2002). Problematizar las prácticas de la enseñanza. Perspectiva, 20(2), 467-482.

Flores, M. A., \& Day, C. (2006). Contexts which shape and reshape new teachers' identities: a multiperspective study. Teaching and Teacher Education, 22(2), 219-232.

Gareis, C. R. (2007). Reclaiming an important teacher competency: the lost art of formative assessment. Journal of Personnel Evaluation in Education, 20, 17-20.

Gelfuso, A., \& Dennis, D. V. (2014). Getting reflection off the page: The challenges of developing support structures for pre-service teacher reflection. Teaching and Teacher Education, 38, 1-11.

Gronlund, N. (2006). Assessment of Student Achievement. Boston: Pearson.

Hammerness, K., Darling-Hammond, L., \& Bransford, J. (2005). How teachers learn and develop. In L. Darling-Hammond \& J. Bransford (Eds.), Preparing teachers for a changing world: What teachers should learn and be able to do (pp.358-389). San Francisco, CA: Wiley \& Sons.

Hamodi, C. (2016). Formar mediante la evaluación en la Universidad. Propuestas útiles para docentes. Valladolid: Universidad de Valladolid.

Higgins, A., Heinz, M., McCauley, V., \& Fleming, M. (2013). Creating the future of teacher education together: the role of emotionality in university-school partnership. Procedia-Social and Behavioral Sciences, 93, 1110-1115.

Hobbs, V. (2011). Reflective practice: Assessing ourselves as teachers. En S. House (Coord.), Inglés. Investigación, innovación y buenas prácticas. Teacher Development (pp. 29-41). Barcelona: Graó.

Kagan, S. (1989). The Structural Approach to Cooperative Learning. Educational Leadership, 47, $12-15$.

Keršyte, N. (2017). Las Ciencias humanas y sociales y la ideología: entre el mal y la necesidad. DeSignis: Publicación de la Federación Latinoamericana de Semiótica (FELS), 26, 31-46

Larrivee, B. (2008). Development of a tool to assess teachers' level of reflective practice. Reflective Practice, 9(3), 341-360.

Lee, H. (2005). Understanding and assessing preservice teachers' reflective thinking. Teaching and Teacher Education, 21, 699-715.

López-Pastor, V. M. (Coord.). (2009). La Evaluación Formativa y Compartida en Docencia Universitaria: propuestas, técnicas, instrumentos y experiencias. Madrid: Narcea.

López-Pastor, V. M. (2011). El papel de la evaluación formativa en la evaluación por competencias: aportaciones de la red de evaluación formativa y compartida en docencia universitaria. Revista de Docencia Universitaria, 9(1), 159-173.

López-Pastor, V. M., \& Pérez-Pueyo, Á. (Coords.). (2017). Evaluación formativa y compartida en educación: experiencias de éxito en todas las etapas educativas. León: Universidad de León, Secretariado de Publicaciones.

Lyons, N. (Comp.). (1999). El uso de portafolios. Propuesta para un nuevo profesionalismo docente. Buenos Aires: Amorrortu Editores.

Martínez, E., \& Raposo, M. (2011). Funciones generales de la tutoría en el Practicum: entre la 
realidad y el deseo en el desempeño de la acción tutorial. Revista de Educación, 354, 155-181.

Martínez, M. C. (2007). El papel del supervisor en las prácticas de enseñanza. Innovación educativa, 17, 137-147.

Molina, E., Bolívar, A., Burgos, A., Domingo, J., Fernández, M., Gallego, M. J., ... Iranzo, P. (2004). La mejora del prácticum, esfuerzo de colaboración. Profesorado, revista de currículum y formación del profesorado, 8(2), 1-31. Recuperado de https://recyt.fecyt.es/index.php/profesorado/article/ view/42153/24107

Montecinos, C., Walker, H., \& Maldonado, F. (2015). School administrators and university practicum supervisors as boundary brokers for initial teacher education in Chile. Teaching and Teacher Education, 49, 1-10.

Pérez, A. I. (1997). Socialización profesional del futuro docente en la cultura de la institución escolar: El mito de las prácticas. Revista Interuniversitaria de Formación del Profesorado, 29, 125-140.

Pérez, M. P. (2005). ¿Se pueden determinar las funciones del supervisor universitario? Revista de Investigación Educativa, 23(2), 315-332.

Pérez, M. P., \& Latorre, M. (2007). Aplicación en el periodo de practicum del modelo reflexivo de supervisión: otra perspectiva del practicum. En M. Iglesias, M. Zabalza, A. Cid y M. Raposo (Coords.), El practicum en el nuevo contexto del Espacio Europeo de Educación Superior. VIII Symposium Internacional sobre el Practicum y las prácticas en empresas en la formación universitaria. Universidade de Santiago de Compostela (pp. 855-868). Recuperado de http:// catalogo.rebiun.org/rebiun/record/Rebiun07181278.

Perrenoud, P. (2004). Desarrollar la práctica reflexiva en el oficio de enseñar. Barcelona: Graó.

Quirk, B., \& Dasovich, J. (1994). The role of supervisor: meeting the needs of early childhood preservice teachers. Paper presented at meeting of the Mid-South Educational Research Association. Nashville. Retrieved from http://files.eric.ed.gov/fulltext/ED388637.pdf.

Rodrigues, P., \& Rodríguez-Illera, J. L. (2014). El portafolio digital como soporte de la práctica reflexiva en la formación docente. Revista Iberoamericana de Educación, 65, 53-74.

Saye, J., \& Brush, T. (2002). Scaffolding critical reasoning about history and social issues in multimedia-supported learning environments. Educational Technology Research and Development, 50, 77-96. Recuperado de https://link.springer.com/article/10.1007/BF02505026

Schön, D. (1983). The reflective practitioner. New York: Basic Books.

Schön, D. (1987). Educating the reflective practitioner. San Francisco: Jossey-Bass Publisher.

Schön, D. (1992). La formación de profesionales reflexivos. Hacia un nuevo diseño de la enseñanza y el aprendizaje en las profesiones. Barcelona: Paidós

Sellars, M. (2012). Teachers and change: The role of reflective practice. Procedia-Social and Behavioural Sciences, 55, 461-469.

Slick, S. (1998). The university supervisor: A disenfranchised outsider. Teaching and Teacher Education, 14(8), 821-834.

Souto, J. M. (2010). ¿Qué escuelas de Geografía para educar en ciudadanía? Didáctica de las ciencias experimentales y sociales, 6, 25-44

Stingu, M. (2012). Reflexive practice in teacher education: facts and trends. Procedia-Social and Behavioural Sciences, 33, 617-621.

Svojanovski, P. (2017). Supporting student teachers' reflection as a paradigm shift process. Teaching and Teacher Education, 66, 338-348.

Ur, P. (1999). A Course in Language Teaching. Practice and Theory. London: Cambridge University Press.

Van Manen, M. (1977). Linking ways of knowing with ways of being practical. Curriculum Inquiry, 6(3), 205-228.

Vilá, R., Aneas, A., \& Rajadell, N. (2015). La evaluación de competencias del alumnado en las Prácticas Externas. La perspectiva de todos los agentes implicados en las Prácticas Externas del grado de Pedagogía de la Universidad de Barcelona. Procedia-Social and Behavioral Sciences, 
Estudios Pedagógicos XLIV, $\mathrm{N}^{\circ}$ 2: 311-328, 2018

EL TUTOR UNIVERSITARIO COMO IMPULSOR DEL APRENDIZAJE REFLEXIVO DE LOS ALUMNOS DURANTE LAS PRÁCTICAS DOCENTES

196, 226-232.

Weber, S. (2013). Can preservice teachers be taught to become reflective thinkers during their first internship experience? PhD Dissertation. Liberty University. Recuperado de http:// digitalcommons.liberty.edu/doctoral/698/.

Zabalza, M. (2011). El Prácticum en la formación universitaria: estado de la cuestión. Revista de Educación, 354, 21-43.

Zabalza, M., \& Cid, A. (2005). Elementos nucleares del Practicum. En M. Raposo, A. Cid, M. Sanmamed, L. Iglesias, M. Muradas y M. A. Zabalza (Coords.), El Practicum en el nuevo contexto del espacio europeo de educación superior. Adenda (pp. 5-22). Santiago de Compostela: Imprenta Universitaria.

Zeichner, K. M. (1990). Educational and Social Commitments in Reflective Teacher Education Programs. In M. Diez (Ed.), Proceedings of the National Forum of the Association of Independent Liberal Arts Colleges for Teacher Education (pp. 55-63). Milwaukee: Alverno College. Recuperado de http://www.academia.edu/25972012/Proceedings_of_the_National_Forum_ of_the_Association_of_Independent_Liberal_Arts_Colleges_for_Teacher_Education_4th_ Milwaukee_Wisconsin_November_9-11_1990_.

Zeichner, K. M. (1992). Formación reflexiva del profesorado desde una perspectiva crítica. En A. Estebaranz y V. Sánchez (Eds.), Pensamiento de profesores y desarrollo profesional (pp. 309326). Sevilla: Servicio de Publicaciones de la Universidad de Sevilla. 